\title{
Infrared to vacuum-ultraviolet ellipsometry and optical Hall-effect study of free-charge carrier parameters in Mg-doped InN
}

\author{
S Schoeche, T Hofmann, Vanya Darakchieva, N Ben Sedrine, X Wang,
}

A Yoshikawa and M Schubert

\section{Linköping University Post Print}

\begin{abstract}
N.B.: When citing this work, cite the original article.
\end{abstract}
Original Publication:

S Schoeche, T Hofmann, Vanya Darakchieva, N Ben Sedrine, X Wang, A Yoshikawa and M Schubert, Infrared to vacuum-ultraviolet ellipsometry and optical Hall-effect study of freecharge carrier parameters in Mg-doped InN, 2013, Journal of Applied Physics, (113), 1, 013502.

http://dx.doi.org/10.1063/1.4772625

Copyright: American Institute of Physics (AIP)

http://www.aip.org/

Postprint available at: Linköping University Electronic Press

http://urn.kb.se/resolve?urn=urn:nbn:se:liu:diva-88457 


\section{AIP Appinied Physics}

\section{Infrared to vacuum-ultraviolet ellipsometry and optical Hall-effect study of free-charge carrier parameters in Mg-doped InN}

S. Schöche, T. Hofmann, V. Darakchieva, N. Ben Sedrine, X. Wang et al.

Citation: J. Appl. Phys. 113, 013502 (2013); doi: 10.1063/1.4772625

View online: http://dx.doi.org/10.1063/1.4772625

View Table of Contents: http://jap.aip.org/resource/1/JAPIAU/v113/i1

Published by the American Institute of Physics.

\section{Related Articles}

Correlations between the morphology and emission properties of trench defects in InGaN/GaN quantum wells J. Appl. Phys. 113, 073505 (2013)

Optical characterization of free electron concentration in heteroepitaxial InN layers using Fourier transform infrared spectroscopy and a $2 \times 2$ transfer-matrix algebra

J. Appl. Phys. 113, 073502 (2013)

Influence of structural anisotropy to anisotropic electron mobility in a-plane InN

Appl. Phys. Lett. 102, 061904 (2013)

Temperature dependent carrier dynamics in telecommunication band InAs quantum dots and dashes grown on InP substrates

J. Appl. Phys. 113, 033506 (2013)

Sub-250nm light emission and optical gain in AIGaN materials

J. Appl. Phys. 113, 013106 (2013)

\section{Additional information on J. Appl. Phys.}

Journal Homepage: http://jap.aip.org/

Journal Information: http://jap.aip.org/about/about_the_journal

Top downloads: http://jap.aip.org/features/most_downloaded

Information for Authors: http://jap.aip.org/authors

\section{ADVERTISEMENT}

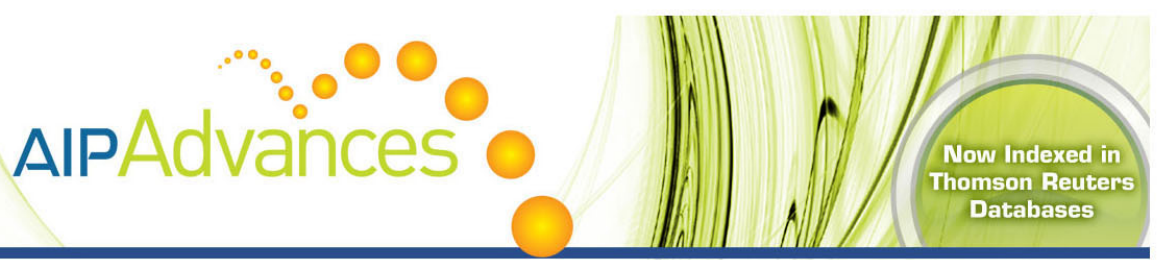

\section{Explore AIP's open access journal: Rapid publication \\ - Article-level metrics \\ Post-publication rating and commenting}




\title{
Infrared to vacuum-ultraviolet ellipsometry and optical Hall-effect study of free-charge carrier parameters in Mg-doped InN
}

\author{
S. Schöche, ${ }^{1, a), b)}$ T. Hofmann, ${ }^{1, b)}$ V. Darakchieva, ${ }^{2}$ N. Ben Sedrine, ${ }^{2,3}$ X. Wang, ${ }^{4}$ \\ A. Yoshikawa, ${ }^{5,6}$ and M. Schubert ${ }^{1, b)}$ \\ ${ }^{1}$ Department of Electrical Engineering, Center for Nanohybrid Functional Materials, University \\ of Nebraska-Lincoln, Lincoln, NE 68588-0511, USA \\ ${ }^{2}$ Department of Physics, Chemistry and Biology (IFM), Linköping University, SE-581 83 Linköping, Sweden \\ ${ }^{3}$ Instituto Tecnológico e Nuclear, 2686-953 Sacavém, Portugal \\ ${ }^{4}$ State Key Laboratory of Artificial Microstructure and Mesoscopic Physics, Peking University, Beijing, China \\ ${ }^{5}$ Center for SMART Green Innovation Research, Chiba University, Chiba, Japan \\ ${ }^{6}$ Department of Information and Communication Engineering, Kogakuin University, Tokyo, Japan
}

(Received 5 September 2012; accepted 29 November 2012; published online 2 January 2013)

\begin{abstract}
Infrared to vacuum-ultraviolet spectroscopic ellipsometry and far-infrared optical Hall-effect measurements are applied to conclude on successful p-type doping of $\mathrm{InN}$ films. A representative set of In-polar $\mathrm{Mg}$-doped $\mathrm{InN}$ films with $\mathrm{Mg}$ concentrations ranging from $1.2 \times 10^{16} \mathrm{~cm}^{-3}$ to $3.9 \times 10^{21} \mathrm{~cm}^{-3}$ is investigated. The data are compared and discussed in dependence of the $\mathrm{Mg}$ concentration. Differences between n-type and p-type conducting samples are identified and explained. p-type conductivity in the $\mathrm{Mg}$ concentration range between $1.1 \times 10^{18} \mathrm{~cm}^{-3}$ and $2.9 \times 10^{19} \mathrm{~cm}^{-3}$ is indicated by the appearance of a dip structure in the infrared spectral region related to a loss in reflectivity of p-polarized light as a consequence of reduced LO phonon plasmon coupling, by vanishing free-charge carrier induced birefringence in the optical Hall-effect measurements, and by a sudden change in phonon-plasmon broadening behavior despite continuous change in the Mg concentration. By modeling the near-infrared-to-vacuum-ultraviolet ellipsometry data, information about layer thickness, electronic interband transitions, as well as surface roughness is extracted in dependence of the $\mathrm{Mg}$ concentration. A parameterized model that accounts for the phonon-plasmon coupling is applied for the infrared spectral range to determine the free-charge carrier concentration and mobility parameters in the doped bulk InN layer as well as the GaN template and undoped InN buffer layer. The optical Hall-effect best-match model parameters are consistent with those obtained from infrared ellipsometry analysis. (C) 2013 American Institute of Physics. [http://dx.doi.org/10.1063/1.4772625]
\end{abstract}

\section{INTRODUCTION}

Recently, InN has attracted substantial research interest due to its potential for applications in high speed electronics, photovoltaics, and optoelectronics. Despite the significant progress in growing high-quality epitaxial InN, control of n-type and p-type conductivity in the material remains challenging. Recently, it was demonstrated that p-type conductivity in $\mathrm{InN}$ can be achieved by $\mathrm{Mg}$-doping. ${ }^{1-7}$ Indications for successful p-type conductivity were only found for $\mathrm{Mg}$ concentrations of $1 \times 10^{18} \mathrm{~cm}^{-3} \leq[\mathrm{Mg}] \leq 3 \times 10^{19} \mathrm{~cm}^{-3}$, which is explained by the necessity to overcome a fairly high intrinsic donor concentration caused by lattice defects and residual shallow donors like hydrogen or oxygen ${ }^{8,9}$ for low $\mathrm{Mg}$ concentrations and an increasing defect density for very high $\mathrm{Mg}$ concentrations. The influence of the polarity of the buffer layer on growth and physical properties of $\mathrm{InN}$ was demonstrated. ${ }^{3,4}$ These results were confirmed by other groups. $^{10}$

The confirmation of successful p-type doping is problematic, mainly due to the observation that the p-type region is

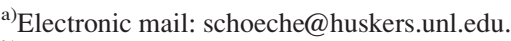

${ }^{\text {b)} U R L: ~ h t t p: / / e l l i p s o m e t r y . u n l . e d u . ~}$
}

embedded in between high-density electron layers that can form at the surface and at the interface between the InN layer and the substrate or the template layer. ${ }^{1,7,11}$ This behavior and the large difference between reported electron mobility parameters $\left(1000-3280 \mathrm{~cm}^{2} / \mathrm{Vs}\right)^{7,12}$ and the estimated hole mobility parameters $\left(20-75 \mathrm{~cm}^{2} / \mathrm{Vs}\right)^{7}$ impede the characterization of the p-type region by conventional electrical methods such as electrical Hall effect or hot probe measurements. ${ }^{13}$ Alternative methods such as electrolyte capacitance-voltage (ECV) measurement, thermo-power, InN-layer thicknessdependent electrical Hall-effect measurements, and variablemagnetic-field electrical Hall-effect measurements were proposed and successfully applied to conclude on the conductivity type and estimate the hole concentration and mobility parameters. $1,7,10,14,15$ Infrared reflectometry (IRR) at normal incidence and mid-IR spectroscopic ellipsometry (IRSE) measurements were demonstrated to be suitable for the analysis of the free-charge carriers in InN. ${ }^{7,11,17-22}$ The advantage of optical methods is the penetration of light through the whole sample stack and the ability to probe underlying layers below the surface accumulation charge. From IRR measurements, the hole concentration and optical mobility parameters of N-polar InN were determined by modeling the contribution of the electron or hole plasma to the dielectric function (DF). 
Different longitudinal optical (LO) phonon-plasmon coupling (LPP) parameters for holes and electrons were obtained due to large differences in effective mass and mobility parameters. ${ }^{11,20-22}$ Normal angle of incidence IRR measurements provide limited sensitivity to electron and hole parameters, especially if additional conducting layers such as a $\mathrm{GaN}$ template layer and an undoped $\mathrm{InN}$ buffer layer are present as in the case for the In-polar InN samples investigated in this study. These additional conducting layers are also contributing to any kind of electrical measurement and are generally hard to separate. An estimation of heavy and light hole mass parameters from acceptor activation energies determined by IRR, IR transmission, and photoluminescence at low temperature was reported recently. ${ }^{21}$ However, the simultaneous and unambiguous determination of hole concentration and mobility parameters by a contactless method remains still a challenge.

In this paper, we present an IRSE and optical Hall-effect (OHE) study in combination with near-IR-vacuum-ultraviolet SE (NIR-VUV-SE) for a set of Mg-doped In-polar InN samples. It is demonstrated how to conclude on p-type conductivity for $\mathrm{Mg}$ concentrations between $1 \times 10^{18} \mathrm{~cm}^{-3}$ and $3 \times 10^{19} \mathrm{~cm}^{-3}$ by combining information gained from the different spectral ranges and techniques. The data are compared and discussed in dependence of the $\mathrm{Mg}$ concentration. Differences between n-type and p-type conducting samples are identified and explained. A parameterized model for the NIR-VUV dielectric function of $\mathrm{InN}$ is developed in order to investigate the influence of the $\mathrm{Mg}$ concentration on the band gap energy and electronic interband transitions. The free-charge carrier parameters concentration and mobility in dependence of the $\mathrm{Mg}$ concentration for the doped InN bulk layer, as well as for the undoped InN buffer layer and the GaN template layer are obtained from analysis of the IRSE data by parameterized dielectric function model analysis that accounts for phonon-plasmon coupling and multiple layers. Best-match model parameters from OHE data analysis are found consistent with those obtained from the IRSE analysis, and will be discussed in view of the InN layer conductivity type.

\section{THEORY}

Spectroscopic ellipsometry is an indirect method and requires a detailed model analysis in order to extract relevant physical parameters. Thus, a stratified layer model analysis using parameterized model DF's is employed to analyze the SE data set. For the samples discussed here, the optical axes of materials constituents, which are optically anisotropic with uniaxial optical properties, is oriented parallel to the surface normal. Therefore, without applying a magnetic field during the measurement, no mode conversion of light polarized parallel (p) to polarization perpendicular (s) to the plane of incidence and vice versa will occur and standard ellipsometry can be applied. ${ }^{23}$ The standard ellipsometric parameters $\Psi$ and $\Delta$ are defined by the ratio $\rho$ of the complex valued Fresnel reflection coefficients

$$
\rho=\frac{r_{p}}{r_{s}}=\tan \Psi \cdot \exp (\mathrm{i} \Delta) .
$$

A common representation of $\Psi$ and $\Delta$ is the pseudo-DF $\langle\varepsilon\rangle$ which is connected to $\rho$ via

$$
\langle\epsilon\rangle=\left[\left(\frac{1-\rho}{1+\rho}\right)^{2} \sin ^{2} \Phi+\cos ^{2} \Phi\right] \tan ^{2} \Phi .
$$

The pseudo-DF is equivalent to the material DF only in the case of a single bulk layer with perfectly smooth surface.

The OHE constitutes ellipsometric measurement of layered semiconducting samples brought into strong external magnetic fields. The generalized ellipsometry formalism is employed. ${ }^{24}$ Generalized ellipsometry determines the dielectric tensor of each sample constituent. The interaction between the electromagnetic plane wave and the sample is conveniently represented using the Mueller matrix calculus. In this formalism, the optical response of the birefringent sample is characterized by the $4 \times 4$ Mueller matrix which relates the real valued Stokes vectors before, $S^{\text {in }}$, and after interaction with a sample: ${ }^{23,25} S^{\text {out }}=M S^{\text {in }}$.

The ellipsometry data were analyzed by using a layerstack model including the sapphire substrate and the GaN template layer. ${ }^{25}$ The light propagation within the entire sample stack is calculated by applying a $4 \times 4$ matrix algorithm for multilayer systems assuming plane parallel interfaces. ${ }^{25}$ In order to reduce parameter correlation, the measurements are obtained at multiple angles of incidence and analyzed simultaneously. A regression analysis (Levenberg-Marquardt algorithm) is performed, where the model parameters are varied until calculated and experimental data match as close as possible. ${ }^{27}$ This is done by minimizing the mean square error $\left(\chi^{2}\right)$ function which is weighed to estimated experimental errors $(\sigma)$. For standard ellipsometry, $\chi_{S t-S E}^{2}$ is calculated by ${ }^{26}$

$$
\chi_{S t-S E}^{2}=\frac{1}{2 S-K} \sum_{i=1}^{S}\left[\left(\frac{\Psi_{i}-\Psi_{i}^{c}}{\sigma_{i}^{\Psi}}\right)^{2}+\left(\frac{\Delta_{i}-\Delta_{i}^{c}}{\sigma_{i}^{\Delta}}\right)^{2}\right],
$$

where the index $i$ indicates the calculated $\left(\Psi_{i}^{c}, \Delta_{i}^{c}\right)$ and experimental $\left(\Psi_{i}, \Delta_{i}\right)$ data pairs at photon energy $E_{i}$ for a total of $S$ single photon energies and $K$ denotes the total number of real valued parameters that are varied during the regression analysis. Accordingly, a weighed mean squared error function $\chi_{G E}^{2}$ for the generalized ellipsometry case can be defined as

$$
\chi_{G E}^{2}=\frac{1}{16 S-K} \sum_{i=1}^{S} \sum_{k=1}^{4} \sum_{l=1}^{4}\left(\frac{M M_{k l, i}-M M_{k l, i}^{c}}{\sigma_{i}^{M M_{k l}}}\right)^{2},
$$

with $M M_{k l, i}$ referring to the elements of the $4 \times 4$ Mueller matrix at the photon energy $E_{i}$.

\section{A. NIR-VUV model dielectric function}

In order to perform the layer model calculations using the $4 \times 4$ matrix algorithm, the DF tensor components of 
each sample constituent are needed as input parameters. For a uniaxial material, the DF tensor is given in diagonal form:

$$
\varepsilon=\left(\begin{array}{ccc}
\varepsilon_{\perp} & 0 & 0 \\
0 & \varepsilon_{\perp} & 0 \\
0 & 0 & \varepsilon_{\|}
\end{array}\right),
$$

with only two different components $\varepsilon_{\perp}$ and $\varepsilon_{\|}$were " $\perp$ " and "\|" denote polarization perpendicular and parallel to the c-axis, respectively. The DF tensor components can be given as tabulated values for each photon energy or can be modeled themselves by using parameterized, energy-dependent algebraic functions, the model dielectric functions (MDF). These MDF describe a specific physical process in the material such as a lattice vibration or an electronic interband transition $^{27}$ and are combined in order to render the overall spectral shape of a material's DF.

The NIR-VUV MDF approaches and parameters of sapphire and $\mathrm{GaN}$ are well established and were taken from databases. ${ }^{28}$ For c-plane InN and measurements performed at angles of incidence between $45^{\circ}$ and $70^{\circ}$, mostly the DF component perpendicular to the c-axis $\varepsilon_{\perp}$ is sensed. ${ }^{16,17}$ The influence of the extraordinary DF component $\varepsilon_{\|}$on the measurement data for this c-plane orientation is small. For determination of this component, measurements on samples of semi-polar or non-polar orientation would be necessary. ${ }^{29}$ Thus, the InN layer was assumed to be isotropic and an effective DF for $\mathrm{InN}$ is obtained for the NIR-VUV spectral range. The isotropic MDF of InN was obtained by using a set of the KramersKronig consistent Herzinger-Johs parameterized semiconductor oscillator models for critical point (CP) structures in crystalline semiconductor DF's (Psemi-CP model). ${ }^{30-32}$ All parameters in this MDF approach were allowed to vary for a undoped reference sample. The shape parameters were then kept fixed and the energy, amplitude, and broadening parameters were varied for each Mg-doped sample separately. To achieve a good agreement between experimental and best-match model calculated data, six Psemi-CP oscillators were included. One oscillator of M0-CP line shape is used for the band gap region. Additionally, five oscillators were necessary to model higher order transitions. A constant offset and a pole function at $11 \mathrm{eV}$ (outside the measured spectral region) for the real part of the MDF are added to account for interband transitions at energies outside the measured spectral range.

The Herzinger-Johs parameterized MDF are designed to perfectly match the DF of arbitrary materials by using Kramers-Kronig consistent oscillator functions while the number of parameters is kept small compared to a point-bypoint analysis. Further, by fixing specific parameters in the model, a specific CP-lineshape can be remodeled and extraction of energies, amplitudes and broadening around a CP is possible. However, due to larger number of parameters compared, for example, to a simple Lorentz oscillator, correlation between the parameters of different oscillators is high and extracted model parameters therefore not unique. In order to determine CP parameters, a widely used approach utilizes analytical line shapes derived from joint density of state calculations of the form:

$$
\varepsilon(E)=C-A \exp (i \Phi)\left(E-E_{0}+\mathrm{i} \Gamma\right)^{\mu},
$$

where $C$ is a constant offset, $A$ is the CP amplitude, $\Phi$ is a phase factor related to the type of $\mathrm{CP}, E_{0}$ is the $\mathrm{CP}$ energy, $\Gamma$ is the CP broadening, and $\mu$ is representing the dimension of the $\mathrm{CP}^{33} \mathrm{Kasic}$ et al. applied this type of analysis in order to describe $\mathrm{CP}$ in n-type InN of different doping levels. ${ }^{17}$ They state that this analysis does not reveal unique results for the dimension of the CP. For this reason, a more basic approach using a sum of simple Lorentz oscillators is applied in this paper

$$
\varepsilon(E)=C+\sum_{j=1}^{n} \frac{A_{n}}{E_{0, n}^{2}-E^{2}+\mathrm{i} \Gamma_{n} E} .
$$

The material DF as determined by applying the HerzingerJohs parameterized model is reanalyzed in the VUV spectral range by using this more simple expression. The extracted parameters should be comparable over the whole sample set with least possible parameter correlation.

The absorption coefficient $\alpha$ of a direct band gap material in dependence of the photon energy $E$ is given by the following equation: ${ }^{34}$

$$
\alpha(E)=A\left(E-E_{g}\right)^{1 / 2} .
$$

The "optical band gap" energy $E_{g}$ is herein defined as the energy above which the square of $\alpha$ increases linearly with increasing photon energy and is extracted by plotting the square of the absorption coefficient in dependence of the photon energy $E$ and determining the interception of a linear fit to this curve with the energy axis. The term "optical band gap" will be used in this paper to emphasize that the determined energy is not equivalent to the electronic band gap energy, i.e., difference between conduction band minimum and valence band maximum, due to doping dependent filling of conduction and valence bands, transition broadening, impurities, exciton-related absorption, and other possible influences. Further, the square root dependence of $\alpha$ on $E$ might only be true in a small vicinity around the optical band gap due to the non-parabolicity of the conduction band of InN. A detailed discussion on how the non-parabolic dispersion of the conduction band influences the optical band gap energy in dependence of the electron concentration in n-type $\mathrm{InN}$ was given by $\mathrm{Wu}$ et al. who used the same approach to determine the optical band gap energy. 35

\section{B. IR model dielectric function}

For the IR spectral range, uniaxial DF with optical axis parallel to the surface normal were assumed for each layer. The sapphire MDF, uncoupled phonon mode parameters, and $\varepsilon_{\infty}$ values for $\mathrm{GaN}$ and $\mathrm{InN}$ were taken from our previous work. ${ }^{18,25,36,38}$ The contribution of free-charge carriers to the MDF and phonon-plasmon coupling was described by applying Kukharskii's model ${ }^{25,37,38}$ 


$$
\varepsilon_{\|, \perp}(\omega)=\varepsilon_{\|, \perp ; \infty} \frac{\left(\omega^{2}+\mathrm{i} \gamma_{\mathrm{LPP}^{-} ; \|, \perp} \omega-\omega_{\mathrm{LPP}^{-} ; \|, \perp}^{2}\right) \cdot\left(\omega^{2}+\mathrm{i} \gamma_{\mathrm{LPP}^{+} ; \|, \perp} \omega-\omega_{\mathrm{LPP}^{+} ; \|, \perp}^{2}\right)}{\omega\left(\omega+\mathrm{i} \gamma_{\mathrm{p} ; \|, \perp}\right) \cdot\left(\omega^{2}+\mathrm{i} \gamma_{\mathrm{TO} ; \|, \perp} \omega-\omega_{\mathrm{TO} ; \|, \perp}^{2}\right)},
$$

where $\omega_{\mathrm{LPP}^{-/+}}$are the frequencies of the $\mathrm{LPP}^{-} / \mathrm{LPP}^{+}$ branches and $\gamma_{\mathrm{LPP}^{-/+}}$is the according LPP broadening parameters. $\gamma_{p}$ is the plasma broadening parameter which is connected to the effective mass $\mathrm{m}^{*}$ and the optical mobility $\mu$ by $\gamma_{\mathrm{p}}=q / m^{*} \mu$, with $|q|=e$ being the elementary charge. $\omega_{\mathrm{LPP}^{-/+}}$can be extracted from

$$
\omega_{\mathrm{LPP}^{-/+}}=\sqrt{\frac{1}{2}\left[\omega_{\mathrm{p}}^{2}+\omega_{\mathrm{LO}}^{2} \pm \sqrt{\left(\omega_{\mathrm{p}}^{2}+\omega_{\mathrm{LO}}^{2}\right)^{2}-4 \omega_{\mathrm{p}}^{2} \omega_{\mathrm{TO}}^{2}}\right]}
$$

with $\omega_{\mathrm{TO} / \mathrm{LO}}$ being the uncoupled $\mathrm{TO} / \mathrm{LO}$ phonon mode frequencies and $\omega_{\mathrm{p}}$ is the plasma frequency given by $\omega_{\mathrm{p}}^{2}=N q^{2} / m^{*} \varepsilon_{0} \varepsilon_{\infty} . N$ is the free-charge carrier concentration. The plasma frequency $\omega_{\mathrm{p}}$ and plasma broadening parameter $\gamma_{\mathrm{p}}$ provide access only to the coupled quantities $\mathrm{N} / \mathrm{m}^{*}$ and $\mu \mathrm{m}^{*}$. Conclusions about carrier concentration and mobility from the IRSE measurements are therefore only possible by assuming a value for the effective mass of electrons or holes, respectively. Further, the sign of the charge $q$ is not accessible without applying an additional magnetic field during the measurement, i.e., direct determination of the carrier type is not possible from IRSE. However, the broadening parameters $\gamma_{\mathrm{LPP}^{-/+}}$are independent of the effective mass of the carriers and directly obtained from the model analysis. Because holes typically reveal much lower mobility and thus higher broadening parameters $\gamma_{\mathrm{LPP}^{-/+}}$than electrons, a different broadening behavior is expected for p-type conducting samples compared to n-type conducting samples and may be taken as indicator of the conductivity type.

\section{Optical Hall-effect model}

The OHE utilizes free-charge carrier related birefringence in an ellipsometry measurement performed with an applied magnetic field. ${ }^{24}$ For the analysis of the OHE data, a sum approach for the MDF of the form $\varepsilon(\omega)=\varepsilon^{\mathrm{L}}(\omega)$ $+\varepsilon^{\mathrm{FC}-\mathrm{MO}}(\omega)$ was applied. The tensor components of the lattice term are described by ${ }^{24}$

$$
\varepsilon_{\|, \perp}^{\mathrm{L}}(\omega)=\varepsilon_{\|, \perp ; \infty} \frac{\omega^{2}+\mathrm{i} \gamma_{\mathrm{LO} ; \|, \perp} \omega-\omega_{\mathrm{LO} ; \|, \perp}^{2}}{\omega^{2}+\mathrm{i} \gamma_{\mathrm{TO} ; \|, \perp} \omega-\omega_{\mathrm{TO} ; \|, \perp}^{2}} .
$$

The free-charge carrier Drude contribution for measurements with applied magnetic field is described by a tensor which allows for the determination of the screened plasma frequency tensor $\omega_{\mathrm{p}}$ and the cyclotron frequency tensor $\omega_{\mathrm{c}}{ }^{25,39}$

$$
\begin{aligned}
& \varepsilon^{\mathrm{FC}-\mathrm{MO}}(\omega)=\omega_{\mathrm{p}}^{2} \\
& \quad \times\left[-\omega^{2} \boldsymbol{I}-i \omega \gamma+i \omega\left(\begin{array}{ccc}
0 & b_{3} & -b_{2} \\
-b_{3} & 0 & b_{1} \\
b_{2} & -b_{1} & 0
\end{array}\right) \omega_{\mathrm{c}}\right]^{-1} .
\end{aligned}
$$

The free-charge carrier mobility tensor is given by $\boldsymbol{\mu}=q /\left(\boldsymbol{\gamma} \boldsymbol{m}^{*}\right)$ where $\boldsymbol{m}^{*}$ denotes the effective mass tensor in units of the free electron mass $m_{0}$ and $\gamma$ is the inverse relaxation time tensor (scattering rate). Effective mass and freecharge carrier mobility are assumed to be isotropic throughout this study, i.e., the according tensors have diagonal form with identical diagonal elements. $\omega_{\mathrm{p}}$ is related to the freecharge carrier density $N$ and the effective mass tensor $\boldsymbol{m}^{*}$ by $\omega_{\mathrm{p}}^{2}=N q^{2} /\left(\varepsilon_{\infty} \tilde{\varepsilon}_{0} \boldsymbol{m}^{*} m_{0}\right)$, where $q$ denotes the charge, $\tilde{\varepsilon}_{0}$ is the vacuum permittivity, and $\varepsilon_{\infty}$ denotes the high frequency dielectric constant. The cyclotron frequency tensor is defined as $\boldsymbol{\omega}_{\mathrm{c}}=q B /\left(m_{0}\right) \boldsymbol{m}^{*-1}$. The external magnetic field for this setup is given by $\boldsymbol{B}=(0,0, B)$, i.e., the external field is oriented perpendicular to the sample surface. Without magnetic field, the magneto-optical tensor $\varepsilon^{\mathrm{FC}-\mathrm{MO}}(\omega)$ reduces to the well known Drude term. Note that the sum approach of the MDF for the analysis of the OHE data does not account for anharmonic phonon-plasma interaction. A magneto-optical DF equivalent to Kukharskii's model is not established yet. Therefore, all OHE measurements presented here were carried out in a spectral range below the LO phonon mode frequencies of InN where the differences between sum approach and Kukharskii model are negligible. The carrier induced birefringence causes mode coupling between $p$ - and s-polarized light. As mentioned before, the generalized ellipsometry formalism needs to be applied for the OHE measurements and the optical response of the sample is represented using the Mueller matrix formalism. ${ }^{25,27}$ Free-charge carrier induced birefringence is indicated by non-zero off-diagonal elements of the Mueller matrix. In our rotating analyzer setup, only the off-diagonal elements $M M_{13}, M M_{23}, M M_{31}$, and $M M_{32}$ are accessible.

\section{EXPERIMENT}

A set of seven samples of $\mathrm{InN}$ films with varying $\mathrm{Mg}$ concentration was grown on Ga-polar c-GaN/sapphire templates by molecular beam epitaxy. The set included an undoped InN reference sample and $\mathrm{Mg}$-doped $\mathrm{InN}$ with systematically increased $\mathrm{Mg}$ concentration ranging from $1.2 \times 10^{16} \mathrm{~cm}^{-3}$ to $8.0 \times 10^{20} \mathrm{~cm}^{-3}$ in order to achieve p-type doping. For all samples, the $\mathrm{InN}$ growth started with a $50 \mathrm{~nm}$ undoped layer on top of which about $400 \mathrm{~nm}$ thick layers of doped $\mathrm{InN}$ were deposited. Yoshikawa et al. demonstrated by ECV measurements that p-type conductivity was achieved for Mg concentrations between $1.1 \times 10^{18} \mathrm{~cm}^{-3}$ and $2.9 \times 10^{19} \mathrm{~cm}^{-3}$ in these samples. ${ }^{7}$

The IRSE measurements have been carried out using a commercial Fourier transform-based IR ellipsometer (J.A. Woollam Co., Inc.) in the spectral range from $300 \mathrm{~cm}^{-1}$ to $6000 \mathrm{~cm}^{-1}$. NIR-VUV measurements were performed on a commercial rotating compensator ellipsometer (NIR-UV) and a commercial nitrogen-purged rotating analyzer ellipsometer (UV-VUV). A custom-built FIR-rotating analyzer 
ellipsometer equipped with a water-cooled magnet that allows for magnetic field amplitudes up to $1.5 \mathrm{~T}$ along the sample normal was used for the FIR OHE measurements. ${ }^{40}$

\section{RESULTS AND DISCUSSION}

\section{A. Investigation on an undoped reference sample}

The influence of the Mg-concentration on the electronic interband transitions is investigated by analyzing ellipsometric data in the IR-VUV spectral range. In order to find an appropriate model for the material DF of $\mathrm{InN}$, the undoped reference sample was investigated first. The measured and best-matching model data for this undoped sample in the IRVUV spectral range are shown in Fig. 1. Measurements on three different ellipsometers have been combined and were analyzed simultaneously. The spectral range below $3.3 \mathrm{eV}$ is plotted on a logarithmic scale in order to emphasize the band gap region. In the IR-NIR spectral range, strong thickness interferences in the $\mathrm{GaN}$ and $\mathrm{InN}$ layers are visible. The frequency of the interference pattern is mostly influenced by the thickness of the GaN template layer while the oscillation of the amplitude is related to the much thinner InN layer. The onset of absorption above the band gap of $\mathrm{InN}$ is indicated by the decrease of the amplitude of the oscillations. With higher photon energies, the interference pattern vanishes because the incoming light is completely absorbed by the InN layer, i.e., only the InN layer is probed in the VUV spectral range.

In order to extract physically relevant parameters, a parameterized critical point model was developed to describe the InN material DF. This model consists of the sapphire substrate, the GaN template layer and the InN bulk layer. Because of minor optical contrast between the undoped InN buffer layer and the doped InN bulk layer in the NIR-VUV spectral range, a single InN layer was also assumed for the doped samples. The surface roughness was taken into

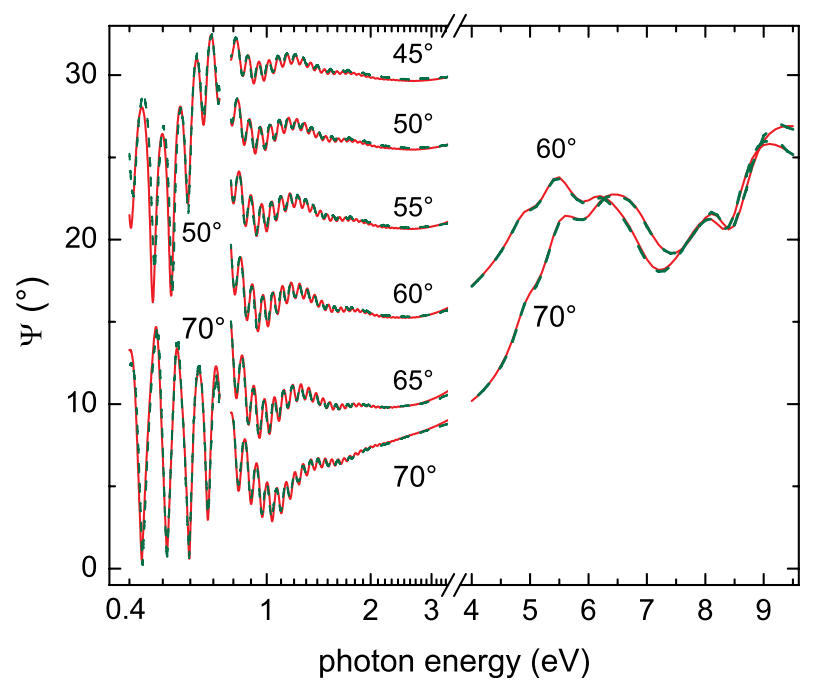

FIG. 1. Experimental (dashed lines) and best-match model calculated (solid lines) $\Psi$-spectra for the undoped reference sample. The spectral range below $3.3 \mathrm{eV}$ is plotted in a logarithmic scale to emphasize the thickness interference pattern within the band gap spectral region. Measurement data from three different instruments are shown. The numerical values indicate the angle of incidence. account by adding a surface layer which was modeled using the Bruggeman effective medium approximation (EMA) by weighing equally the $\mathrm{DF}$ of $\mathrm{InN}$ and air. ${ }^{27}$ The excellent agreement between model and experiment shown in Fig. 1 could be achieved by using six Herzinger-Johs oscillators to describe the critical points in $\mathrm{InN}$ in the spectral range $0.4 \mathrm{eV}$ to $9.5 \mathrm{eV}$. This model corroborates the results found by other authors for undoped or n-type conductive $\mathrm{InN}$ on different substrates. ${ }^{17,29,41}$ To label the critical points, the nomenclature of Goldhahn et l $^{41}$ is used here. A M0-CP lineshape is utilized for the band gap $\left(\mathrm{E}_{0}\right)$ region. M1-CP lineshapes are used for the transitions $\mathrm{A}, \mathrm{E}_{1}, \mathrm{E}_{2}$, and $\mathrm{E}_{4}$. The critical point structure $\mathrm{E}_{3}$ was modeled by using a M2-CP lineshape. The resulting material $\mathrm{DF}$ for the undoped $\mathrm{InN}$ film is shown in a comparison with the doped samples in Fig. 4 in Sec. IV B.

The strongest influence of free-charge carriers on the DF is typically seen in the IR spectral range in form of freecharge carrier related absorption and interaction with phonons. The experimental and best-match model calculated $\Psi$-data for the Mid-IR spectral range of the undoped InN sample are shown in Fig. 2. The position of the uncoupled phonon modes of E- and A-symmetry for each material are indicated by brackets, where dashed lines mark TO phonons and solid lines are indicating the uncoupled LO phonon. The spectrum is dominated by features that can be assigned to the reststrahlen bands of the sapphire substrate and the $\mathrm{GaN}$ template layer. The features below $470 \mathrm{~cm}^{-1}$ are related to sapphire phonon modes. The $\mathrm{A}_{1}-\mathrm{TO}$ phonon of $\mathrm{InN}$ is not influencing the spectrum. ${ }^{42}$ The dip at $479 \mathrm{~cm}^{-1}$ is caused by the onset of the reststrahlen band in $\mathrm{InN}$ at the $\mathrm{E}_{1}$-TO frequency. The InN layer is not thick enough to completely absorb the incoming light. Thus, a dip-like structure at $510 \mathrm{~cm}^{-1}$ is present in the spectra caused by a loss in preflectivity of the sapphire substrate. ${ }^{36}$ Above the $\mathrm{E}_{1}$-TO mode frequency at $560 \mathrm{~cm}^{-1}$, the GaN template layer becomes totally reflecting, which can be seen as an increase in $\Psi$. The small loss in reflectivity of p-polarized light

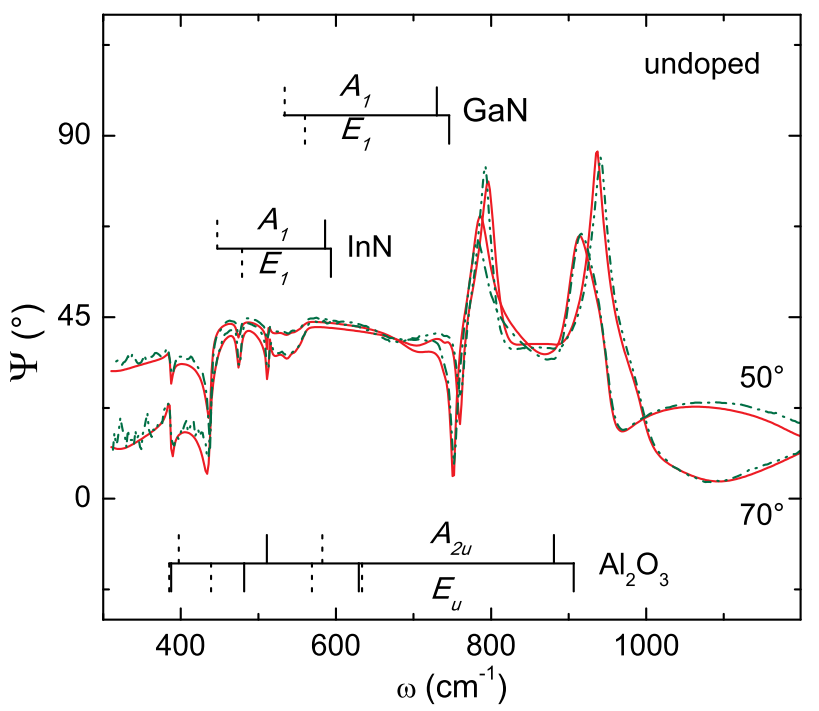

FIG. 2. Same as Fig. 1 for the mid-IR spectral region. The uncoupled phonon mode energies are indicated by markers for TO (dashed) and LO (solid) phonons for each of the sample constituents. 
around $700 \mathrm{~cm}^{-1}$ is caused by the $\mathrm{A}_{1} \mathrm{LPP}^{+}$mode of $\mathrm{InN}$ that as shifted compared to the uncoupled $\mathrm{A}_{1}$ - $\mathrm{LO}$ mode due to a high background electron concentration in this sample. ${ }^{17}$ The strong dip at about $750 \mathrm{~cm}^{-1}$ is related to reduced reflectivity of the GaN buffer layer beyond the reststrahlen band that ends at the $\mathrm{E}_{1}-\mathrm{LO}$ mode of $\mathrm{GaN}$ at $745 \mathrm{~cm}^{-1}$ for an undoped GaN layer. This dip is slightly shifted to the higher energy of about $755 \mathrm{~cm}^{-1}$ due to the existence of a small number of electrons in the GaN layer. The peak structure towards higher energy is caused by thickness interferences inside the $\mathrm{GaN}$ and $\mathrm{InN}$ layers in a spectral range where the substrate is still totally reflecting. The shape of these structures is related to the anisotropy of the $\mathrm{GaN}$ and $\mathrm{InN}$ as indicated by $\Psi$ values above $45^{\circ}$. The peak maxima shift with the free-charge carrier parameters in the $\mathrm{GaN}$ and $\mathrm{InN}$ buffer layers and allow for determination of the free charge carrier parameters of the $\mathrm{GaN}$ template and the $\mathrm{InN}$ buffer layer. The interferences end above the reststrahlen band of the sapphire substrate.

In the IR-VUV spectral range, the $50 \mathrm{~nm}$ buffer layer cannot be separated from the bulk InN films due to the marginal difference in the material DF. In contrast, a strong influence of the free-charge carrier related contribution to the DF in the IR spectral in form of additional absorption (Drude term) and shift of phonon mode frequencies due to LPP coupling allows for separate determination of freecharge carrier parameters in the undoped buffer and doped InN bulk layer. Accordingly, in the IR spectral range the $\mathrm{InN}$ was modeled following the growth recipe as consisting of a bulk InN layer and a buffer layer with a fixed thickness of $50 \mathrm{~nm}$. The buffer layer also accounts for possible accumulation of free-charge carriers at the interface between $\mathrm{GaN}$ template and InN. The thickness values of the GaN template layer and the total thickness of the $\mathrm{InN}$ layers as obtained from the IR-VUV measurements were used as input parameters for the IRSE analysis. In order to achieve the demonstrated agreement between model and experimental data, we allowed for variation of the free-charge carrier parameters in the GaN template layer, the $\mathrm{InN}$ buffer layer and the $\mathrm{InN}$ bulk layer. The parameters for $\mathrm{GaN}$ and the $\mathrm{InN}$ buffer layer were assumed to be identical for all samples and thus coupled during a multiple sample analysis. Isotropically averaged mobilities and effective masses were used for all layers and accordingly LPP broadening values for the DF components parallel to the optical axis (A-modes) were assumed to be the same as for the DF components perpendicular to the optical axis (E-modes). The influence of a surface accumulation layer on the IRSE spectra was reported to be small due to a strong $\mathrm{LPP}^{+}$broadening. ${ }^{11,17}$ An attempt to additionally model a surface accumulation charge revealed that high surface electron concentration in a very thin layer on top of the bulk InN layer would mainly influence the position and peak height of the interference pattern above $800 \mathrm{~cm}^{-1}$. This structure could be modeled well by only allowing for free-charge carriers in the $\mathrm{GaN}$ and $\mathrm{InN}$ buffer layers. The additional surface layer caused higher parameter correlation without significant improvement of the modelexperiment match. Fixing the free-charge carrier parameters of the accumulation layer throughout the whole sample set did not seem to be suitable due to the significant change of the structural properties and free-charge carrier properties of the InN layers with increasing $\mathrm{Mg}$ concentration. In conclusion, we neglect the influence of this layer in our IRSE analysis. We note, however, that IRSE data on non-c-plane oriented samples and/or InN grown directly on sapphire as well as SE measurements in magnetic fields (OHE) provide enough sensitivity to determine the surface charge sheet density. ${ }^{18,19,43}$ For the GaN template layer an electron concentration of $2.9 \times 10^{17} \mathrm{~cm}^{-3}$ and a mobility of $102 \mathrm{~cm}^{2} / \mathrm{Vs}$ was obtained under assumption of an effective mass of $0.23 m_{0}$ for $\mathrm{GaN}^{38}$ Using the dependence of the electron effective mass in $\mathrm{InN}$ on the carrier concentration as determined by $\mathrm{OHE}^{43}$ a buffer layer electron concentration of $1.1 \times 10^{19} \mathrm{~cm}^{-3}$ and a mobility of $132 \mathrm{~cm}^{2} / \mathrm{Vs}$ were determined.

As mentioned in Sec. II, IRSE cannot provide direct evidence for p-type conductivity. Thus, additional FIR-OHE measurements, i.e., SE measurements with applied magnetic field, were performed in order to prove the p-type conductivity. Using the OHE, it is in general possible to determine the free-charge carrier parameters concentration, mobility, and effective mass independently as well as the type of carrier (holes or electrons). ${ }^{24,39}$ Further, parameters of free-charge carriers in different layers of a heterostructure can be determined individually. ${ }^{24,43,44}$ Figure 3 shows the experimental and best-match model calculated data of OHE measurements performed on the undoped reference sample in the FIR spectral range at an angle of incidence of $72^{\circ}$. The differences of spectra taken at magnetic field strengths of $+1.5 \mathrm{~T}$ and $-1.5 \mathrm{~T}$ for the off-diagonal Mueller-matrix elements $M M_{31}$ and $M M_{32}$ are shown. By plotting difference spectra, influences of intrinsic anisotropy or slight sample misalignment are eliminated and only the free-charge carrier related parts of the spectra are remaining. The main characteristic feature in this spectral range is a peak structure around the $\mathrm{A}_{1}-\mathrm{TO}$

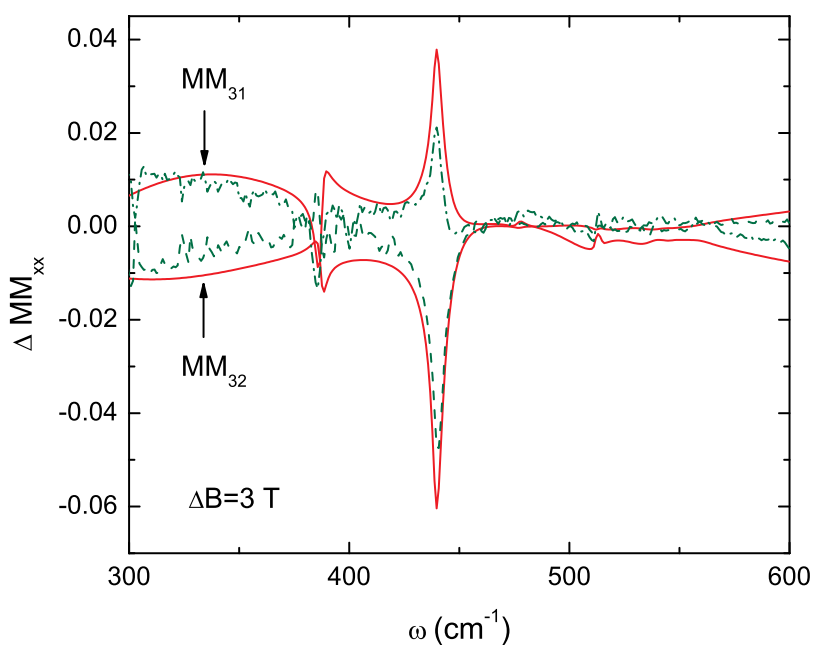

FIG. 3. Experimental (dotted lines) and best-match calculated (solid lines) data of FIR-OHE measurements performed at an angle of incidence of $72^{\circ}$ for the undoped reference sample. The differences of spectra taken at magnetic field strengths of $+1.5 \mathrm{~T}$ and $-1.5 \mathrm{~T}$ of the off-diagonal Mueller-matrix elements $M M_{31}$ and $M M_{32}$ are plotted. For the model calculated data, freecharge carrier parameters as determined by the IRSE analysis were used without further regression analysis. 

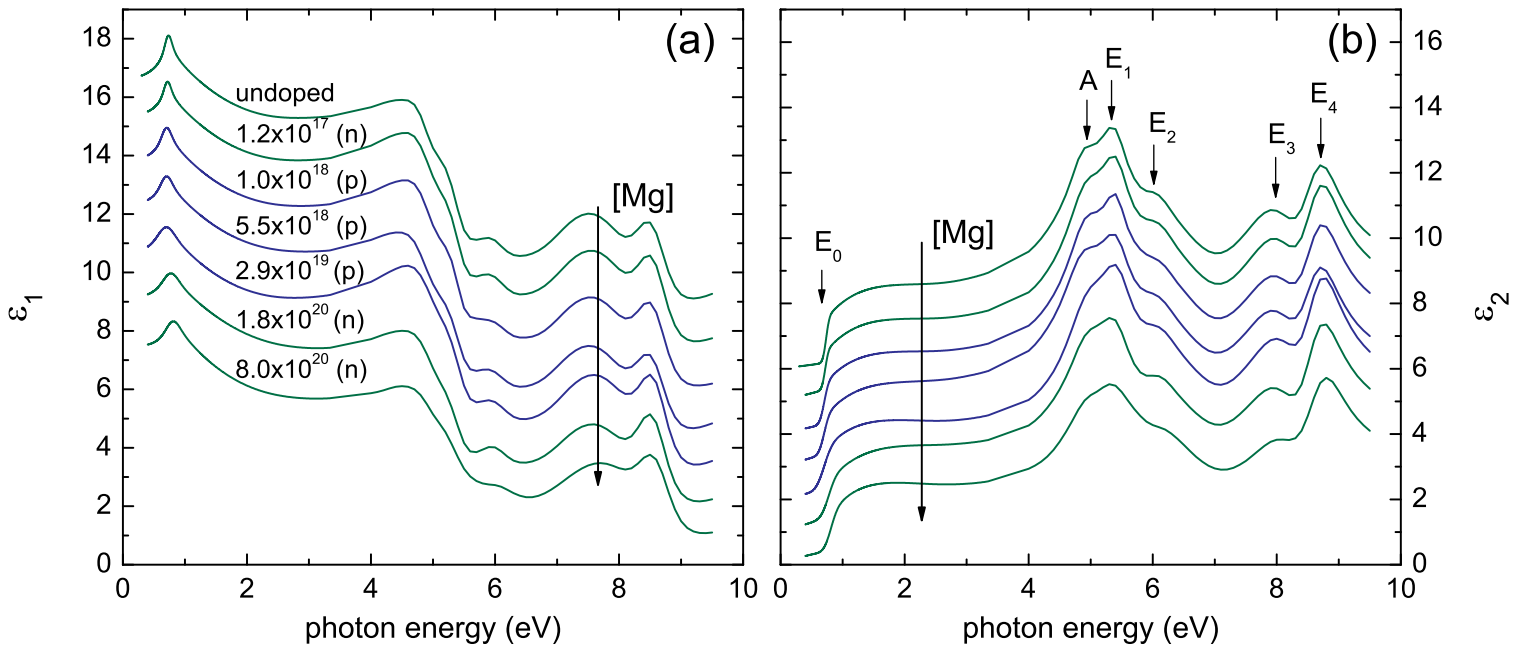

FIG. 4. Real (a) and imaginary (b) part of the MDF of InN for different Mg concentrations as extracted from a parameterized oscillator model for the data shown in Fig. 1. The symbols in parenthesis indicate electron (n) or hole (p) conductivity. The position of critical points labeled according to Goldhahn $e t$ al. ${ }^{41}$ are indicated by arrows. An offset of 1.5 in (a) and 1 in (b) is added between the data sets for clarity.

mode of InN at $440 \mathrm{~cm}^{-1}$. The model data shown in the graph are simulated by using the free-charge carrier parameters extracted from IRSE without further regression analysis. The excellent match between experimental and best-model calculated data proves the validity of the IRSE results.

\section{B. Results for Mg-doped InN samples}

Figure 4 shows the real and imaginary part of the bestmatching MDF for different Mg concentrations as extracted from the parameterized Herzinger-Johs model analysis in the NIR-VUV spectral range. In order to extract CP parameters and be able to compare these parameters between samples of different Mg concentrations, the best-match MDF was reanalyzed using a sum of five Lorentz oscillators. If limited only to the VUV spectral range, a very good fit between experimental and model data can be achieved. The resulting experimental and best-match model data for the imaginary part of the pseudo-DF is shown in Fig. 5. The energetic position of

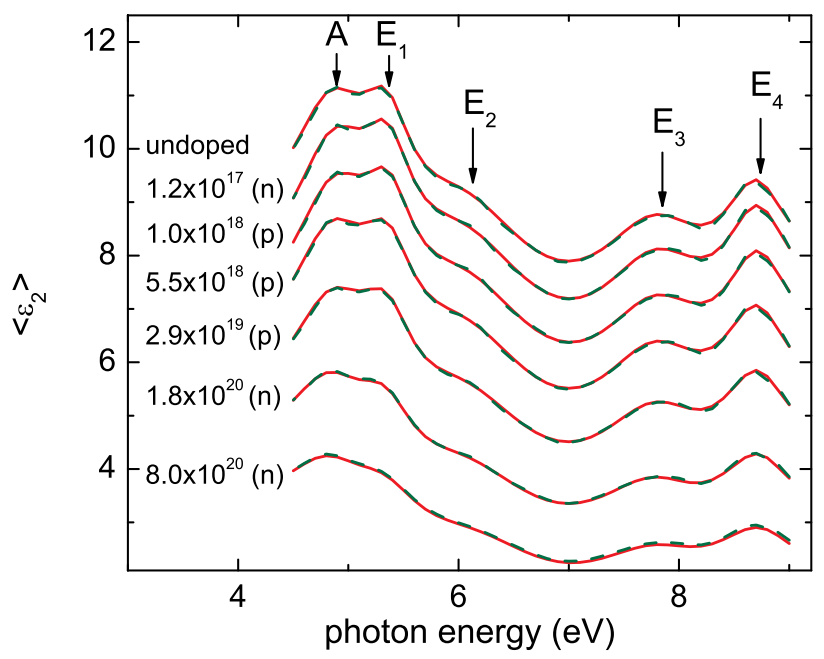

FIG. 5. Experimental (dashed lines) and best-matching model (solid lines) of $\left\langle\varepsilon_{2}\right\rangle$. Data for an angle of incidence of $70^{\circ}$ are shown. The positions of critical points labeled according to Goldhahn et al. ${ }^{41}$ are indicated by arrows. An offset of 0.8 added between the data sets for clarity. each CP structure is indicated by arrows. Goldhahn et al. found $\mathrm{CP}$ structures at $4.88 \mathrm{eV}, 5.35 \mathrm{eV}, 6.05 \mathrm{eV}, 7.87 \mathrm{eV}$, and $8.6 \mathrm{eV}$ for the ordinary component of not intentionally doped MBE-grown InN films on GaN buffer with a-plane and c-plane orientation. ${ }^{29,41}$ The electron concentration as determined from electrical Hall-effect for their c-plane sample was $1.5 \times 10^{18} \mathrm{~cm}^{-3}$ which is similar to the electron concentration in our undoped sample (see IRSE results in Fig. 14). The energetic position of the CP structures obtained in our study agree very well with the values given by Goldhahn et al. (Fig. 6). We find no systematic dependence of the interband transitions above $4 \mathrm{eV}$ on the $\mathrm{Mg}$ concentration or conductivity type. The amplitudes of the CP $A, E_{1}$, and $E_{2}$ are slightly correlated as expected for overlapping oscillator functions which results in larger error bars compared to the $\mathrm{CP} E_{3}$ and $E_{4}$. However, an overall decreasing absorption

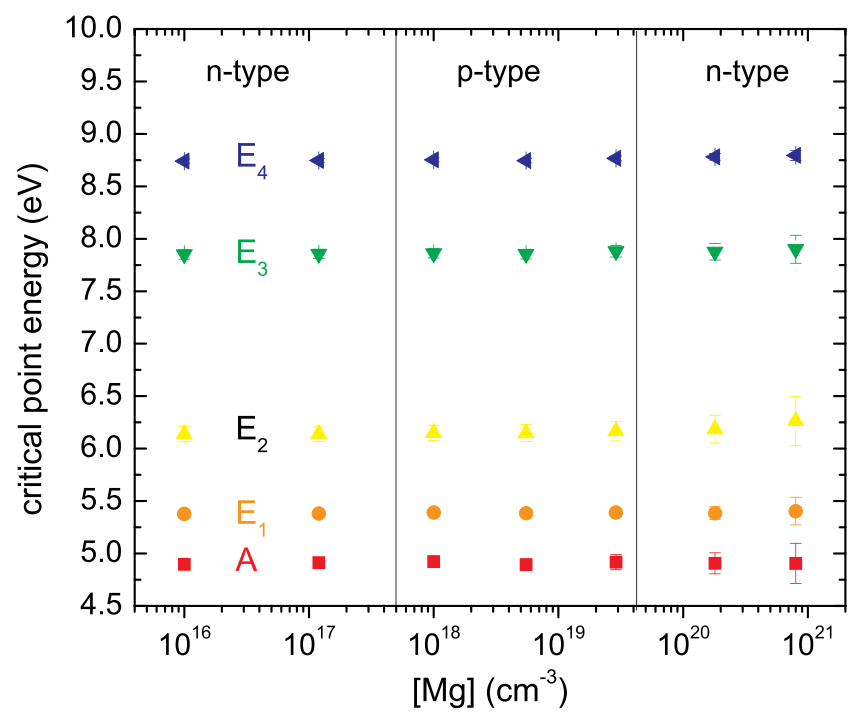

FIG. 6. Energetic position of the critical points in $\mathrm{InN}$ for different $\mathrm{Mg}$ concentrations as determined from the parameterized model analysis. The undoped sample parameters are given at a $\mathrm{Mg}$ concentration of $10^{16} \mathrm{~cm}^{-3}$. The change of conductivity type as determined by Yoshikawa et al. ${ }^{7}$ is indicated by vertical lines. 
strength is indicated by a decrease of the amplitude of the $\mathrm{CP}$ oscillators for $\mathrm{Mg}$ concentrations above $1.8 \times 10^{20} \mathrm{~cm}^{-3}$ (Fig. 7), i.e., the n-type samples of very high electron concentrations (compare to IRSE results in Fig. 14). Further, a slight increase of the broadening parameters of the $\mathrm{CP}$ oscillators for $\mathrm{Mg}$ concentration above $1.8 \times 10^{20} \mathrm{~cm}^{-3}$ is found (Fig. 8). This behavior can be explained by higher defect densities for increasing $\mathrm{Mg}$ concentration. In accordance, increasing surface roughness values for $\mathrm{Mg}$ concentrations above $2.9 \times 10^{19} \mathrm{~cm}^{-3}$ are determined (Table I). Even though the absolute thickness values for the roughness layer differ from the roughness values determined by using atomic force microscopy (AFM), similar trend is found. The discrepancy between the SE and AFM values might be explained by averaging over a larger area in the SE measurements and by deviations from a 50:50 ratio between void and material as assumed in the EMA approach for the surface layer. An increase of the surface roughness layer thickness from about $3 \mathrm{~nm}$ for all samples with $[\mathrm{Mg}] \leq 1 \times 10^{19} \mathrm{~cm}^{-3}$ to much higher values for the highly doped samples is determined from the SE analysis. Yoshikawa et al. confirmed that this drastic increase of the surface roughness for high $\mathrm{Mg}$ concentrations is caused by a change in growth mode. ${ }^{7}$

In contrast to the higher order interband transitions, a significant dependence of the band gap energy value on the dopant concentration was reported for n-type $\mathrm{InN}$ by several authors. ${ }^{17,35,41,45}$ While the intrinsic band gap of $\mathrm{InN}$ at room temperature should be around $0.60 \mathrm{eV},{ }^{45}$ band gap renormalization effects and Burstein-Moss shift due to partial filling of the conduction band lead to an increased experimentally determined band gap energy. ${ }^{17,35,41,45}$ A detailed discussion of the individual mechanisms that cause this shift and how it is influenced by the non-parabolic dispersion of the conduction band in $\mathrm{InN}$ can be found in the paper by $\mathrm{Wu}$ et al. ${ }^{35}$ In order to examine the value of the optical band gap energy in dependence of the $\mathrm{Mg}$ concentration, the absorption coefficient $\alpha$ was calculated from the best matching

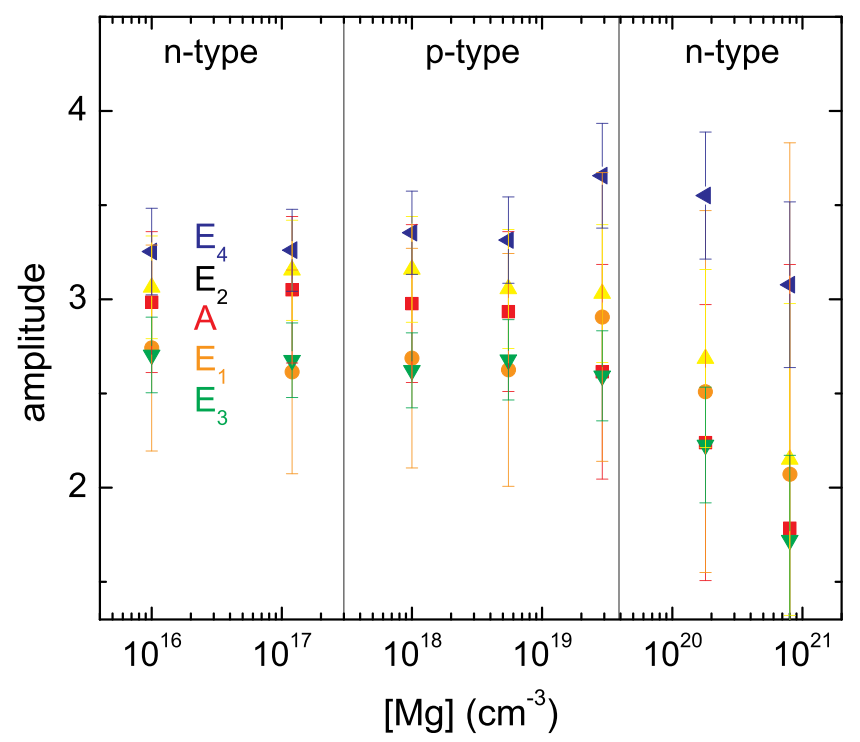

FIG. 7. Best-match model amplitude values for the $\mathrm{CP}$ oscillators for different $\mathrm{Mg}$ concentration as obtained by a parameterized model analysis. The undoped sample parameters are given at a $\mathrm{Mg}$ concentration of $10^{16} \mathrm{~cm}^{-3}$.

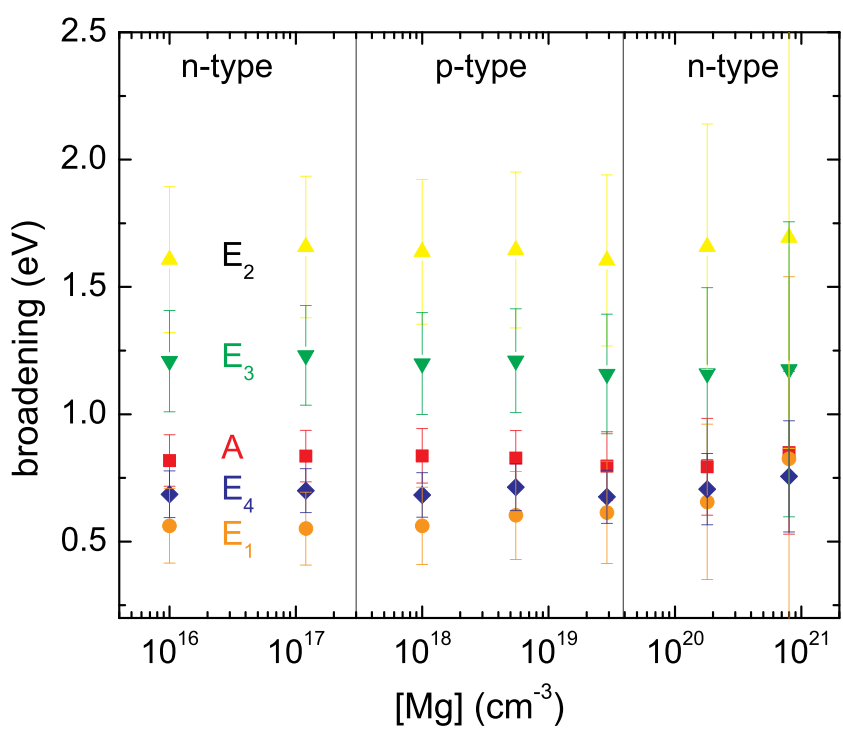

FIG. 8. Best-match model broadening values for the CP oscillators for different $\mathrm{Mg}$ concentration as obtained by a parameterized model analysis. The undoped sample parameters are given at a $\mathrm{Mg}$ concentration of $10^{16} \mathrm{~cm}^{-3}$.

Herzinger-Johs parameterized model analysis and the square of it was plotted in dependence of the photon energy. The value of the optical band gap energy can then be approximated by determining the intercept of a linear fit to this curve with the energy axis. Figure 9 shows these plots and the determined optical band gap energy values in dependence of the Mg concentration. A slight decrease of the optical band gap energy value compared to the undoped sample might occur for $\mathrm{Mg}$ concentrations up to $2.9 \times 10^{19} \mathrm{~cm}^{-3}$. These changes are too small to allow for conclusions on the influence of the $\mathrm{Mg}$ concentration or free-charge carrier type on the optical band gap energy. However, a systematic blueshift of the optical band gap energy is indicated for higher $\mathrm{Mg}$ concentrations. The determined optical band gap energy values match the values determined by $\mathrm{Wu}$ et al. and Kasic et al. for n-type samples of the same free-charge carrier concentration and plasma frequencies as determined in our set for $\mathrm{Mg}$ concentrations above $2.9 \times 10^{19} \mathrm{~cm}^{-3}$ (plasma frequencies for our samples are given in Fig. 11). ${ }^{17,35}$

The comparison of the experimental and best-matching model IRSE data in dependence of the $\mathrm{Mg}$-concentration is presented in Fig. 10. All measurements show similar spectral

TABLE I. InN layer thickness, surface roughness as determined by the parameterized Herzinger-Johs model analysis (SE roughness), and RMS value determined from AFM. The sample with a $\mathrm{Mg}$ concentration of $[\mathrm{Mg}]=1.2 \times 10^{17} \mathrm{~cm}^{-3}$ was not included in the AFM analysis.

\begin{tabular}{lccc}
\hline \hline$[\mathrm{Mg}]\left(\mathrm{cm}^{-3}\right)$ & $\begin{array}{c}\text { InN layer thickness } \\
(\mathrm{nm})\end{array}$ & $\begin{array}{c}\text { SE roughness } \\
(\mathrm{nm})\end{array}$ & $\begin{array}{c}\text { AFM RMS } \\
(\mathrm{nm})\end{array}$ \\
\hline undoped & 454.8 & 3.2 & 1.0 \\
$1.2 \times 10^{17}$ & 440.8 & 2.5 & $\ldots$ \\
$1.1 \times 10^{18}$ & 459.5 & 3.0 & 0.9 \\
$5.5 \times 10^{18}$ & 448.2 & 3.0 & 1.2 \\
$2.9 \times 10^{19}$ & 418.9 & 5.2 & 1.3 \\
$1.8 \times 10^{20}$ & 444.0 & 7.3 & 8.1 \\
$8.0 \times 10^{20}$ & 440.3 & 9.6 & 13.5 \\
\hline \hline
\end{tabular}




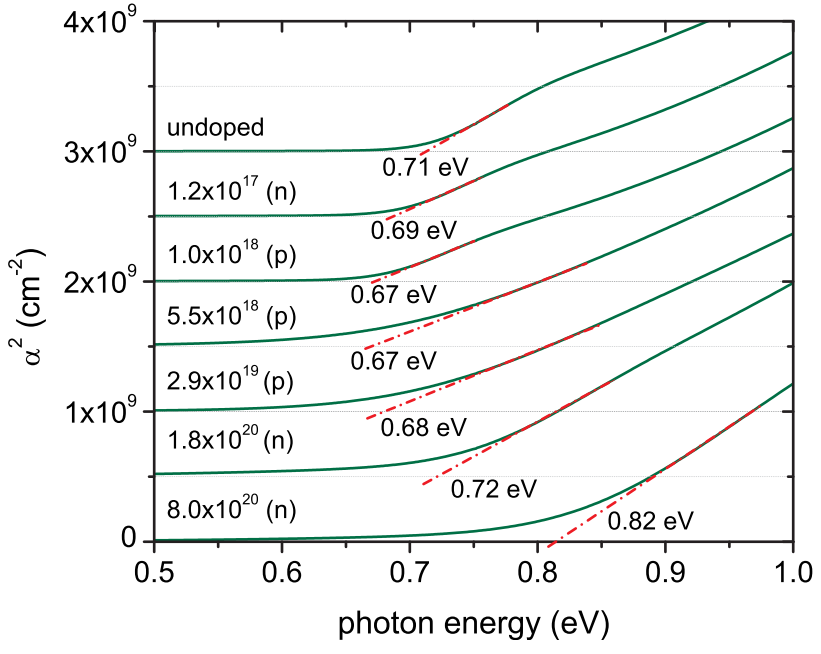

FIG. 9. Square of the absorption coefficient $\alpha$ in dependence of the photon energy $E$ for different $\mathrm{Mg}$ concentrations in the spectral range near the optical band gap of InN. The intercept of a linear fit to the curve is assumed to represent the optical band gap energy value of the InN layer. The determined values are given at the point of interception. Curves are offset by $5 \times 10^{8} \mathrm{~cm}^{-2}$ for clarity.

features as the undoped sample and can be explained in the same way. The most obvious difference of the samples that are supposed to be p-type conductive compared to the n-type samples is the existence of a dip that occurs slightly above the uncoupled $\mathrm{A}_{1}-\mathrm{LO}$ mode of $\mathrm{InN}$ at around $600 \mathrm{~cm}^{-1}$. This

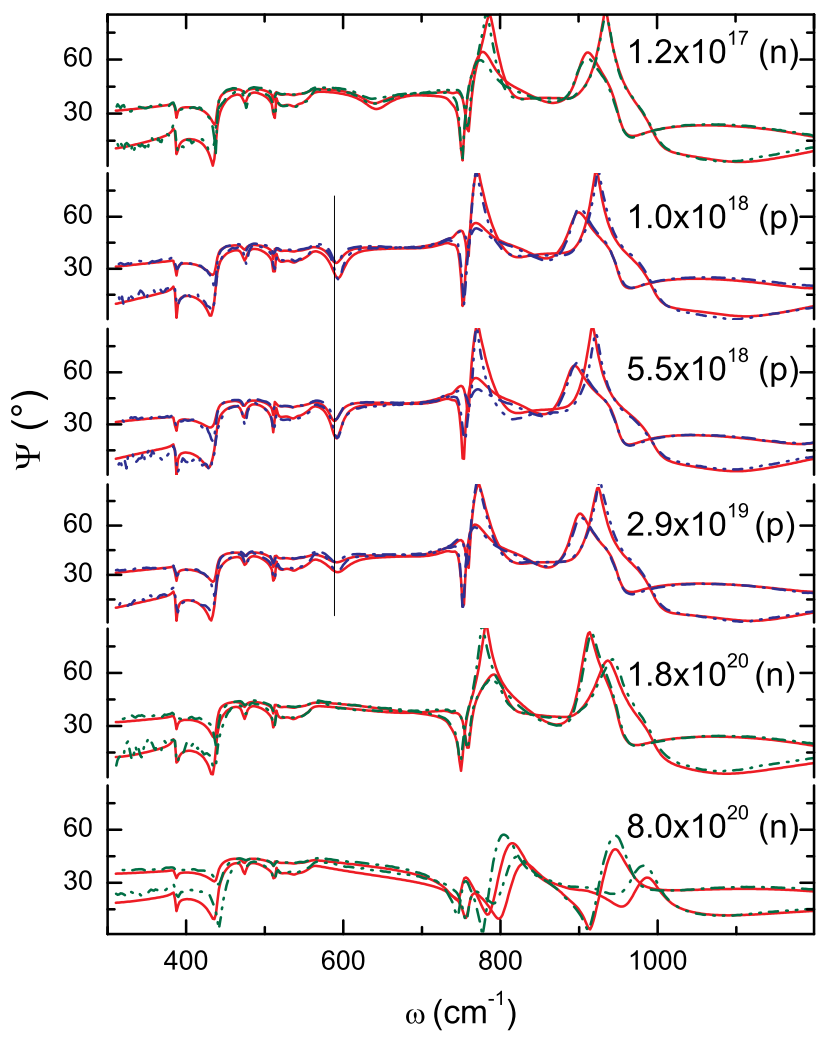

FIG. 10. Comparison of experimental (dashed lines) and best-matching model data (solid lines) of IRSE measurements for samples of different $\mathrm{Mg}$ concentration. The characteristic dip around $600 \mathrm{~cm}^{-1}$ due to the reduced LPP shift and broadening of the InN $\mathrm{A}_{1}$-LPP mode for the p-type conducting samples is marked by the vertical line.

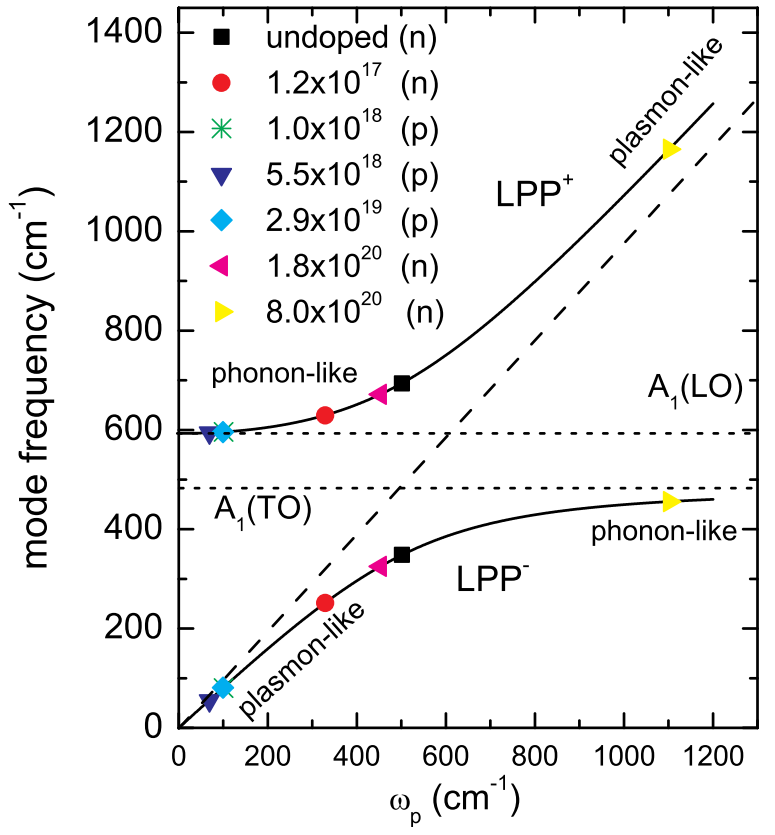

FIG. 11. LPP branches in dependence of the plasma frequency $\omega_{\mathrm{p}}$. The position of the $\mathrm{LPP}^{+/-}$modes is indicated by symbols for each sample as obtained from the model analysis.

dip is caused by a reduced reflectivity of p-polarized light and can be explained by the strongly reduced LPP coupling of the $\mathrm{A}_{1}$-LPP mode due to much higher hole effective mass and very small mobility of holes compared to electrons. Due to reduced LPP coupling these modes are close to the uncoupled $\mathrm{A}_{1}$-LO of $\mathrm{InN}$, however a remaining slight shift indicates the presence of free-charge carriers in these samples. Accordingly, much lower values for the plasma frequency are obtained from the model analysis for the p-type samples compared to all n-type samples (Fig. 11). The spectral position of the dip is mainly influenced by the freecharge carrier concentration, while the depth is determined by the broadening parameter $\gamma_{\mathrm{LPP}^{+}}$.

As explained in Sec. II, the LPP broadening parameters are independent parameters in the model for the IRSE analysis and might therefore also indicate a change in free-charge carrier type since different broadening behavior is expected for holes and electrons. Figure 12 shows the broadening values $\gamma_{\mathrm{LPP}^{+/-}}$for the upper and lower LPP branches as directly obtained from the regression analysis. Despite continuous increasing of the $\mathrm{Mg}$ concentration a sudden change in the broadening parameters (logarithmic scale) is observed for the $\mathrm{Mg}$ concentration range between $1.1 \times 10^{18} \mathrm{~cm}^{-3}$ and $2.9 \times 10^{19} \mathrm{~cm}^{-3}$. Below this concentration range, broadening parameters of equal magnitude are determined with $\gamma_{\mathrm{LPP}^{+}}$ being slightly smaller than $\gamma_{\mathrm{LPP}^{-}}$. For the very high doped $n-$ type samples, the broadening value $\gamma_{\mathrm{LPP}^{+}}$of the upper LPP mode is significantly higher than the broadening value $\gamma_{\mathrm{LPP}^{-}}$ of the lower LPP mode. This agrees well with the expected plasmon-like behavior of the $\mathrm{LPP}^{+}$mode for high electron concentrations. ${ }^{38}$ Within the n-type conducting samples, a general increase of the LPP broadening values is observed, which can be associated to structural degradation with increasing $\mathrm{Mg}$ concentration and corroborates the results obtained from the NIR-VUV analysis. We believe that the 


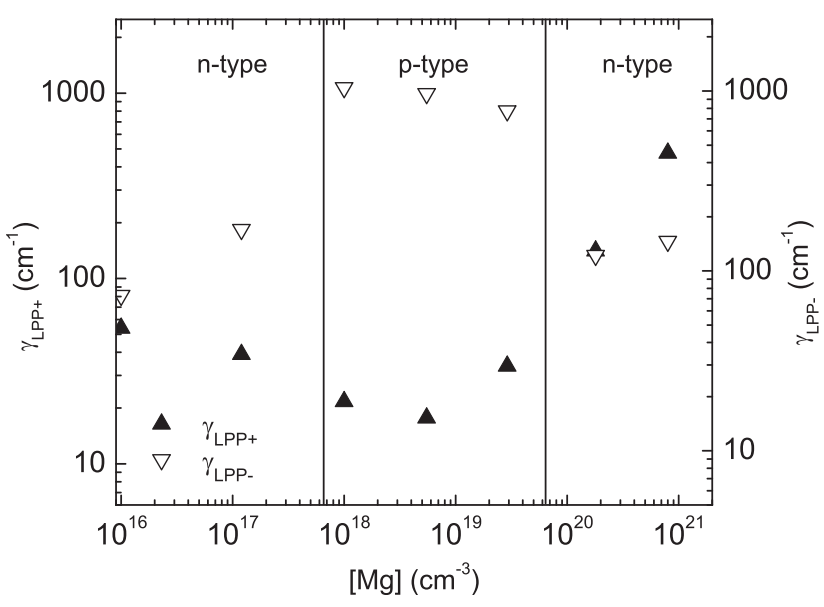

FIG. 12. Best-match model parameters for the LPP broadening parameters as obtained from the regression analysis. Values for the undoped reference sample are indicated on the coordinate axis on the left at $[\mathrm{Mg}]=1 \times 10^{16} \mathrm{~cm}^{-3}$.

sudden change in the broadening behavior, that is found for the $\mathrm{Mg}$ concentration range mentioned above, despite the continuous change of $\mathrm{Mg}$ concentration, validates the assumption that these LPP broadening parameters are associated with hole conductivity. Additionally, an attempt to model the IRSE data for this $\mathrm{Mg}$ concentration range by using effective mass parameter values for electrons in InN resulted in very small mobility values below $150 \mathrm{~cm}^{2} / \mathrm{Vs}$. This result seems unreasonable compared to mobility values determined for the n-type samples of even higher dopant concentrations above $2.9 \times 10^{19} \mathrm{~cm}^{-3}$ (see Fig. 14) and supports our assumption.

The existence of a different free-charge carrier type for the samples of $\mathrm{Mg}$ concentrations between $1.1 \times 10^{18} \mathrm{~cm}^{-3}$ and $2.9 \times 10^{19} \mathrm{~cm}^{-3}$ is further indicated by the OHE data. The comparison of the difference spectra of the off-diagonal Mueller-matrix element $M M_{31}$ in dependence of the $\mathrm{Mg}$ concentration is shown in Fig. 13. The magnitude of the peak structure around $440 \mathrm{~cm}^{-1}$ is strongly reduced in case of the p-type conducting samples. This reduced free-charge carrier-induced birefringence indicates the absence of electrons in the bulk InN layer for $\mathrm{Mg}$ concentrations between $1.1 \times 10^{18} \mathrm{~cm}^{-3}$ and $2.9 \times 10^{19} \mathrm{~cm}^{-3}$. Due to the very high effective mass and low mobility of holes in p-type InN, the hole related birefringence is found to be very small. The hole related contribution to the experimental data is further overlapped by strong birefringence related to highly mobile electrons in the GaN template layer and the contribution of electrons in the InN buffer layer and the surface accumulation layer. Thus, an extraction of the hole parameters from the OHE data for the sample set used in this study turned out to be impossible at the available magnitude and orientation of the magnetic field of only $1.5 \mathrm{~T}$ along the surface normal. However, the experimental data support the results obtained by IRSE and NIR-VUV. We see from the IRSE analysis that free-charge carriers are present. Since the experimental OHE does not show electron related birefringence, it seems reasonable to suppose that these carriers must be holes. To support the results from the IRSE analysis, model data are also

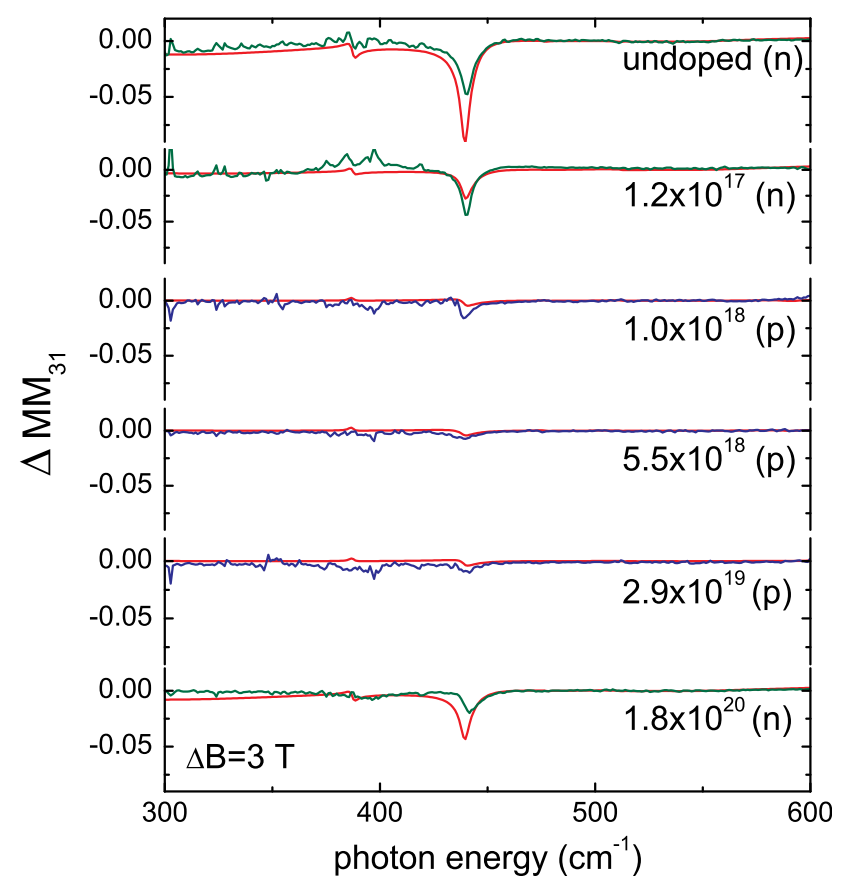

FIG. 13. Experimental and best-match model data of FIR-OHE measurements performed at an angle of incidence of $72^{\circ}$. The difference of spectra taken at magnetic field strengths of $+1.5 \mathrm{~T}$ and $-1.5 \mathrm{~T}$ of the off-diagonal Mueller-matrix element $M M_{31}$ is plotted.

shown in Fig. 13. As for the undoped reference sample, this model data were simulated by using the free-charge carrier parameters as determined in the IRSE analysis without further regression analysis. Slight differences between model and experimental data are very likely related to the neglect of the surface accumulation layer. Higher magnetic fields and an optimized reflection geometry might allow to determine all hole parameters, including the effective mass, and would also be required to determine surface accumulation charge parameters from a regression analysis of the OHE data.

\section{Discussion of the resulting free-charge carrier parameters}

In conclusion, $\mathrm{p}$-type conductivity in the $\mathrm{Mg}$ concentration range $1.1 \times 10^{18} \mathrm{~cm}^{-3}$ and $2.9 \times 10^{19} \mathrm{~cm}^{-3}$ is indicated by the appearance of a dip structure in the IRSE spectra related to a loss in reflectivity of p-polarized light as consequence of reduced LPP coupling, by vanishing free-charge carrier induced birefringence in the OHE measurements, and by the sudden change in LPP broadening behavior despite continuous change in the $\mathrm{Mg}$ concentration. Assuming an effective hole mass of $0.42 m_{0}$ for the p-type samples ${ }^{11}$ and using the dependence of the electron effective mass on the carrier concentration as determined by $\mathrm{OHE},{ }^{43}$ it is therefore now possible to deduce the parameters of the free-charge carrier concentration $N$ and the mobility $\mu$ from $\omega_{\mathrm{p}}$ and $\gamma_{\mathrm{p}}$, which is plotted in Fig. 14.

The principle behavior of carrier concentration $N$ and mobility $\mu$ is similar to the results obtained for $\mathrm{N}$-polar $\mathrm{p}-\mathrm{InN}$ samples. ${ }^{11,21}$ However, slightly smaller values for the carrier density and mobility of our In-polar p-type InN samples are 


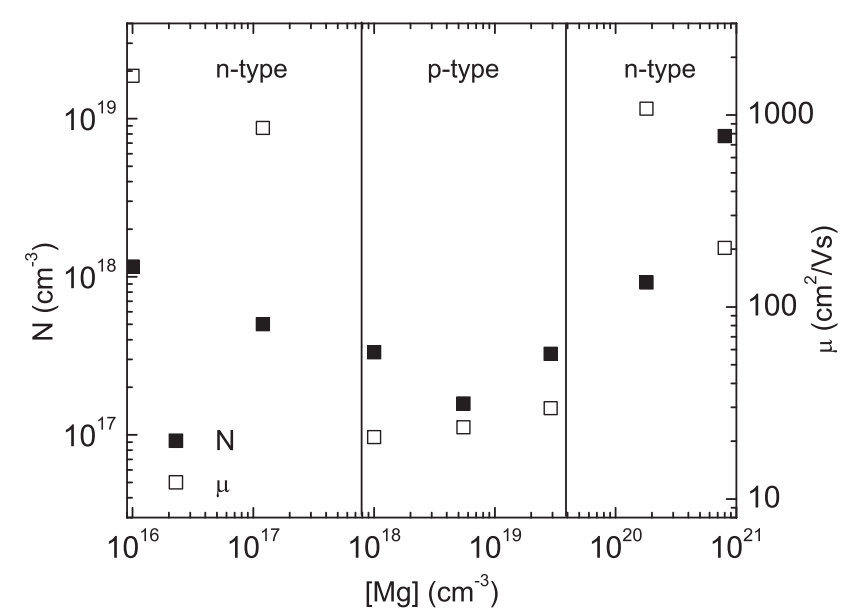

FIG. 14. Parameter values for the free-charge carrier concentration $N$ and mobility $\mu$ as determined from the IRSE model analysis. Values for the undoped reference sample are indicated on the coordinate axis on the left at $[\mathrm{Mg}]=1 \times 10^{16} \mathrm{~cm}^{-3}$.

observed. A systematic change of the free-charge carrier concentration with increasing $\mathrm{Mg}$ concentration is observed. For low $\mathrm{Mg}$ concentration of $1.2 \times 10^{17} \mathrm{~cm}^{-3}$ a reduction of the electron concentration compared to the undoped sample is found, which can be explained by partial compensation of residual background donors. For higher $\mathrm{Mg}$ concentrations, the number of acceptor atoms exceeds the number of background donors, the sample becomes p-type conductive. ${ }^{7}$ The hole density in the p-type samples does not systematically increase with increasing $\mathrm{Mg}$ concentration. Instead, we find a reduction of the hole density when increasing the $\mathrm{Mg}$ concentration from $1.1 \times 10^{18} \mathrm{~cm}^{-3}$ to $5.5 \times 10^{18} \mathrm{~cm}^{-3}$. A further increase of the $\mathrm{Mg}$ concentration to $2.9 \times 10^{19} \mathrm{~cm}^{-3}$ gives a similar hole concentration as for the $\mathrm{Mg}$ concentration of 1.1 $\times 10^{18} \mathrm{~cm}^{-3}$. For $[\mathrm{Mg}] \geq 2.9 \times 10^{19} \mathrm{~cm}^{-3}$ defect induced donors over-compensate the acceptors and the samples become n-type. This observation could be related to results by Yoshikawa et al. who reported a switch of polarity and increasing surface roughness values for $[\mathrm{Mg}] \geq 1$ $\times 10^{19} \mathrm{~cm}^{-3}$. The increase of the surface roughness layer thickness as shown in Table I from about $3 \mathrm{~nm}$ for all samples with $[\mathrm{Mg}] \leq 1 \times 10^{19} \mathrm{~cm}^{-3}$ to significantly higher values for larger $\mathrm{Mg}$ concentration supports this finding.

Except for the highest $\mathrm{Mg}$ concentration of 8.0 $\times 10^{20} \mathrm{~cm}^{-3}$, mobilities over $1000 \mathrm{~cm}^{2} / \mathrm{Vs}$ are found for the n-type conductive samples. The p-type conductive samples show mobility values between $22 \mathrm{~cm}^{2} / \mathrm{Vs}$ and $29 \mathrm{~cm}^{2} / \mathrm{Vs}$. It should be noted that even when assuming the effective masses to be on the order of the effective electron mass, the obtained mobility values would still be one order of magnitude smaller than the electron mobilities in the n-type samples, which also indicates a different carrier type in these samples. Our results fit very well to the average hole mobility of $23 \mathrm{~cm}^{2} / \mathrm{Vs}$ determined by Wang et al..$^{5}$ by estimating sheet conductivities from electrical Hall-effect measurements on samples with different $\mathrm{InN}$ bulk layer thicknesses and of $19 \mathrm{~cm}^{2} / \mathrm{Vs}$ determined by thermopower measurements by Bierwagen et al. ${ }^{15}$ Larger hole mobility values of up to $70 \mathrm{~cm}^{2} /$ Vs obtained by FTIR reflectometry on N-polar samples were reported by Fujiwara et al. ${ }^{11}$ However, separation of contributions of additional conducting layers to the reflectometry data taken at normal incidence is difficult. In contrast, ellipsometry measurements at multiple oblique angles of incidence provide the ability to separate contributions from underlaying layers from the bulk InN layer properties. $^{25}$

\section{SUMMARY AND CONCLUSION}

In summary, we applied IRSE in combination with FIROHE and NIR-VUV SE measurements to study free-charge carrier properties in In-polar InN samples that were systematically doped with $\mathrm{Mg}$ to achieve p-type conductivity. A complete parameterized model analysis over the wide spectral range from $300 \mathrm{~cm}^{-1}(0.037 \mathrm{eV})$ up to $9.5 \mathrm{eV}$ was performed to extract free-charge carrier parameters as well as information about interband transitions. By combining the different spectral ranges and techniques, the existence of a bulk p-type layer can be concluded from comparing the data in dependence of the $\mathrm{Mg}$ concentration. A parameterized model of Herzinger-Johs oscillators describing CP lineshapes for the NIR-VUV spectral range is derived to extract thickness values, surface roughness layer thickness and best matching material DF for the InN layer. A CP analysis using a sum of Lorentz oscillators is performed in order to determine comparable CP energy, amplitude, and broadening values for the VUV spectral range. The onset of absorption in dependence of the $\mathrm{Mg}$ concentration is determined by analyzing absorption spectra calculated from the Herzinger-Johs best match model. A blue-shift of the optical band gap energy for $\mathrm{Mg}$ concentrations above $2.9 \times 10^{19} \mathrm{~cm}^{-3}$ is found in accordance with results found by other authors for highly doped n-type samples. A decrease in structural quality for $\mathrm{Mg}$ concentrations above $2.9 \times 10^{19} \mathrm{~cm}^{-3}$ manifests in a significant increase in surface roughness, decreasing absorption strength and increased broadening of the interband transitions. A Mg concentration dependent shift of higher $\mathrm{CP}$ energies could not be observed. In the IR spectral range, the well-established Kukharskii model is applied to account for the interaction of the free-charge carriers with LO phonons to form LO phonon plasmons. Reduced LPP-coupling, seen as a characteristic dip in the IRSE data, and qualitatively different broadening behavior for $\mathrm{Mg}$ concentrations between $1.1 \times 10^{18} \mathrm{~cm}^{-3}$ and $2.9 \times 10^{19} \mathrm{~cm}^{-3}$ indicate the existence of a p-type conducting bulk InN layer for these $\mathrm{Mg}$ concentrations. The absence of electrons in the p-type InN films is demonstrated as vanishing free-charge carrier induced birefringence in additional OHE measurements which support the conclusion of a change in the conductivity type from n-type to p-type conductivity for the $\mathrm{Mg}$ concentration range found from the IRSE analysis. By applying established values for the effective mass of electrons in GaN, using the non-parabolic band model for the effective mass of electrons in InN, and assuming a effective hole mass of $0.42 m_{0}$ for holes in InN, the concentration and mobility values of the free-charge carriers in the GaN-template layer, the undoped InN buffer layer, and the $\mathrm{Mg}$-doped $\mathrm{InN}$ bulk layer as well as the LPP broadening values were determined in 
dependence of the $\mathrm{Mg}$ concentration. However, direct access to all hole parameters, including the effective mass, could not be achieved yet. The results obtained in this study go along with results obtained by other authors by combining several different techniques and allow to conclude that IRSE in combination with $\mathrm{OHE}$ is a very suitable technique to inspect conductivity type and free-charge carrier parameters in InN films.

\section{ACKNOWLEDGMENTS}

The authors acknowledge financial support from the National Science Foundation under Award Nos. MRSEC DMR-0820521, MRI DMR-0922937, DMR-0907475, and EPS-1004094, by the Swedish Research Council (VR) under Grant No. 2010-3848, the Swedish Governmental Agency for Innovation Systems (VINNOVA) under the VINNMER International Qualification program, Grant No. 2011-03486 and by FCT Portugal under contract PTDC/FIS/100448/ 2008, and program Ciência 2007.

${ }^{1}$ R. E. Jones, K. M. Yu, S. X. Li, W. Walukiewicz, J. W. Ager, E. E. Haller, H. Lu, and W. J. Schaff, Phys. Rev. Lett. 96, 125505 (2006).

${ }^{2}$ X. Wang, S. Che, Y. Ishitani, and A. Yoshikawa, Appl. Phys. Lett. 90, 201913 (2007).

${ }^{3}$ X. Wang, S. Che, Y. Ishitani, A. Yoshikawa, H. Sasaki, T. Shinagawa, and S. Yoshida, Appl. Phys. Lett. 91, 081912 (2007).

${ }^{4}$ X. Wang, S. Che, Y. Ishitani, and A. Yoshikawa, Appl. Phys. Lett. 91, 242111 (2007).

${ }^{5}$ X. Wang, S. Che, Y. Ishitani, and A. Yoshikawa, Appl. Phys. Lett. 92, 132108 (2008)

${ }^{6}$ X. Wang, G. Zhao, Q. Zhang, Y. Ishitani, A. Yoshikawa, and B. Shen, Appl. Phys. Lett. 96, 061907 (2010).

${ }^{7}$ A. Yoshikawa, X. Wang, Y. Ishitani, and A. Uedono, Phys. Status Solidi A 207, 1011 (2010)

${ }^{8}$ C. S. Gallinat, G. Koblmüller, and J. S. Speck, Appl. Phys. Lett. 95, 022103 (2009).

${ }^{9}$ V. Darakchieva, K. Lorenz, N. P. Barradas, E. Alves, B. Monemar, M. Schubert, N. Franco, C. L. Hsiao, W. J. Schaff, L. W. Tu, T. Yamaguchi, and Y. Nanishi, Appl. Phys. Lett. 96, 081907 (2010).

${ }^{10}$ K. Wang, N. Miller, R. Iwamoto, T. Yamaguchi, M. A. Mayer, T. Araki, Y. Nanishi, K. Yu, E. E. Haller, W. Walukiewicz et al., Appl. Phys. Lett. 98, 042104 (2011).

${ }^{11}$ M. Fujiwara, Y. Ishitani, X. Wang, S. Che, and A. Yoshikawa, Appl. Phys. Lett. 93, 231903 (2008).

${ }^{12}$ X. Wang, S. Liu, N. Ma, L. Feng, G. Chen, F. Xu, N. Tang, S. Huang, K. J. Chen, S. Zhou, and B. Shen, Appl. Phys. Express 5, 015502 (2012).

${ }^{13}$ W. Keenan, C. Schneider, and C. Pillius, Solid State Technol. 14, 51 (1971).

${ }^{14}$ P. A. Anderson, C. H. Swartz, D. Carder, R. J. Reeves, S. M. Durbin, S. Chandril, and T. H. Myers, Appl. Phys. Lett. 89, 184104 (2006).

${ }^{15}$ O. Bierwagen, S. Choi, and J. Speck, Phys. Rev. B 85, 165205 (2012).

${ }^{16}$ D. Aspnes, JOSA 70, 1275 (1980).
${ }^{17}$ A. Kasic, E. Valcheva, B. Monemar, H. Lu, and W. J. Schaff, Phys. Rev. B 70, 115217 (2004).

${ }^{18}$ V. Darakchieva, T. Hofmann, M. Schubert, B. E. Sernelius, B. Monemar, P. Persson, F. Giuliani, E. Alves, H. Lu, and W. J. Schaff, Appl. Phys. Lett. 94, 022109 (2009).

${ }^{19}$ V. Darakchieva, M. Schubert, T. Hofmann, B. Monemar, C.-L. Hsiao, T.-W. Liu, L.-C. Chen, W. J. Schaff, Y. Takagi, and Y. Nanishi, Appl. Phys. Lett. 95, 202103 (2009).

${ }^{20}$ Y. Ishitani, X. Wang, S. Che, and A. Yoshikawa, J. Appl. Phys. 103, 053515 (2008).

${ }^{21}$ M. Fujiwara, Y. Ishitani, X. Wang, K. Kusakabe, and A. Yoshikawa, J. Appl. Phys. 110, 093505 (2011).

${ }^{22}$ Y. Ishitani, M. Fujiwara, X. Wang, S. Che, and A. Yoshikawa, Phys. Status Solidi C 6, S397 (2009).

${ }^{23} \mathrm{M}$. Schubert, in Handbook of Ellipsometry, edited by E. A. Irene and H. G. Tompkins (William Andrew, 2004).

${ }^{24}$ T. Hofmann, C. Herzinger, and M. Schubert, Phys. Status Solidi A 205, 779 (2008).

${ }^{25}$ M. Schubert, Infrared Ellipsometry on Semiconductor Layer Structures: Phonons, Plasmons and Polaritons, Springer Tracts in Modern Physics Vol. 209 (Springer, Berlin, 2004).

${ }^{26}$ C. M. Herzinger, B. Johs, W. A. McGahan, J. A. Woollam, and W. Paulson, J. Appl. Phys. 83, 3323 (1998).

${ }^{27}$ H. Fujiwara, Spectroscopic Ellipsometry: Principles and Applications (John Wiley \& Sons Ltd., 2007).

${ }^{28}$ J. A. Woollam Co., Inc., Spectroscopic Ellipsometry Software WVASE32 ${ }^{\circledR}$.

${ }^{29}$ R. Goldhahn, A. Winzer, V. Cimalla, O. Ambacher, C. Cobet, W. Richter, N. Esser, J. Furthmüller, F. Bechstedt, H. Lu et al., Superlattices Microstruct. 36, 591 (2004).

${ }^{30}$ J. A. Woollam Co., Inc., Guide to Using WVASE32.

${ }^{31}$ B. Johs, C. M. Herzinger, J. H. Dinan, A. Cornfeld, and J. D. Benson, Thin Solid Films 313, 137 (1998).

${ }^{32} \mathrm{C}$. Herzinger and B. Johs, "Dielectric function parametric model, and method of use," U.S. patent 5,796,983 (18 August 1998).

${ }^{33}$ P. Lautenschlager, M. Garriga, L. Vina, and M. Cardona, Phys. Rev. B 36, 4821 (1987).

${ }^{34}$ J. Pankove, Optical Processes in Semiconductors (Dover, 1971).

${ }^{35}$ J. Wu, W. Walukiewicz, W. Shan, K. M. Yu, J. W. Ager, E. E. Haller, H. Lu, and W. J. Schaff Phys. Rev. B 66, 201403 (2002).

${ }^{36}$ M. Schubert, T. E. Tiwald, and C. M. Herzinger, Phys. Rev. B 61, 8187 (2000).

${ }^{37}$ A. A. Kukharskii, Solid State Commun. 13, 1761 (1973).

${ }^{38}$ A. Kasic, M. Schubert, S. Einfeldt, D. Hommel, and T. E. Tiwald, Phys. Rev. B 62, 7365 (2000).

${ }^{39}$ M. Schubert, T. Hofmann, and C. M. Herzinger, J. Opt. Soc. Am. A 20 , 347 (2003).

${ }^{40}$ T. Hofmann, C. M. Herzinger, J. L. Tedesco, D. K. Gaskill, J. A. Woollam, and M. Schubert, Thin Solid Films 519, 2593 (2011).

${ }^{41}$ R. Goldhahn, P. Schley, A. T. Winzer, M. Rakel, C. Cobet, N. Esser, H. Lu, and W. J. Schaff, J. Cryst. Growth 288, 273 (2006).

${ }^{42}$ V. Darakchieva, P. P. Paskov, E. Valcheva, T. Paskova, B. Monemar, M. Schubert, H. Lu, and W. J. Schaff, Appl. Phys. Lett. 84, 3636 (2004).

${ }^{43}$ T. Hofmann, V. Darakchieva, B. Monemar, H. Lu, W. J. Schaff, and M. Schubert, J. Electron. Mater. 37, 611 (2008).

${ }^{44}$ T. Hofmann, A. Boosalis, P. Kühne, C. M. Herzinger, J. A. Woollam, D. K. Gaskill, J. L. Tedesco, and M. Schubert, Appl. Phys. Lett. 98, 041906 (2011).

${ }^{45}$ W. Walukiewicz, J. W. Ager, K. M. Yu, Z. Liliental-Weber, J. Wu, S. X. Li, R. E. Jones, and J. D. Denlinger, J. Phys. D: Appl. Phys. 39, R83 (2006). 